\title{
"Ferveu, queimou o ser da erva": conhecimentos de especialistas locais sobre plantas medicinais na região Sul do Brasil
}

\author{
BALDAUF, C. ${ }^{1^{*}}$; KUBO, R.R. ${ }^{2}$; SILVA, F. ${ }^{3}$; IRGANG, B.E. ${ }^{4}$ \\ ${ }^{2}$ Universidade Estadual de Campinas, Programa de Pós-Graduação em Biologia Vegetal. Endereço: PPG-BV/ \\ Instituto de Biologia da Unicamp, Cidade Universitária Zeferino Vaz, s/n, Caixa Postal 6109, Barão Geraldo, Campinas/ \\ SP, CEP 13083-970. * crisbaldauf@yahoo.com.br ${ }^{2}$ Universidade Federal do Rio Grande do Sul, Núcleo de \\ Estudos em Desenvolvimento Rural Sustentável em áreas de Mata Atlântica (DESMA-UFRGS). Endereço: Av. \\ João Pessoa, 41, Centro, Porto Alegre/RS, CEP 90040-000. E-mail: rumikubo@bol.com.br ${ }^{3}$ Universidade Federal \\ de Santa Catarina, Programa de Pós-Graduação em Agroecossistemas. Endereço: Centro de Ciências Agrárias, \\ Rodovia Admar Gonzaga, 1346, Bairro Itacorubi, CEP 88034-001 Florianópolis, SC, Brasil. E-mail: \\ pavitrii2005@yahoo.com.br ${ }^{4}$ In memoriam
}

\begin{abstract}
RESUMO: O objetivo desta pesquisa foi realizar levantamento etnobotânico sobre o conhecimento e uso de plantas medicinais por especialistas locais na comunidade do Lami, situada na região sul de Porto Alegre, Rio Grande do Sul. A metodologia envolveu a aplicação de entrevistas semiestruturadas e excursões guiadas. Para cada citação as seguintes informações foram registradas: nome botânico e vernacular, uso terapêutico, parte da planta utilizada, preparação e forma de administração. Foi calculado o valor de uso e a porcentagem de concordância quanto aos usos principais corrigida para cada planta citada, bem como o fator de consenso entre informantes para as diferentes categorias de doenças consideradas. Registrou-se o conhecimento sobre 141 espécies medicinais, pertencentes a 63 famílias botânicas. O conhecimento dos especialistas é bastante amplo e inclui o uso de plantas nativas e exóticas quase na mesma proporção. As plantas medicinais representam uma importante alternativa terapêutica na comunidade estudada. O uso associado das medicinas tradicional e convencional, bem como as formas de transmissão e conservação do conhecimento são também discutidos neste artigo.
\end{abstract}

Palavras-chave: etnobotânica, medicina popular, Porto Alegre, conhecimento local

\begin{abstract}
Ferveu, queimou o ser da erva": knowledge of local experts on medicinal plants in Southern Brazil. The aim of this study was to conduct an ethnobotanical survey focusing on the knowledge and use of medicinal plants by local experts from Lami community, located in the south region of Porto Alegre, Rio Grande do Sul, Brazil. The methodology involved semi-structured interviews and guided excursions. For each citation the following data were recorded: botanical and vernacular name, therapeutic use, part used, preparation and administration form. The use value was calculated, as well as the corrected percentage of agreement concerning the main uses for each cited plant and the factor of consensus among informants for the different categories of the considered diseases. Knowledge about 141 medicinal species of 63 botanical families was recorded. The experts have an extensive knowledge including the use of native and exotic plants almost at the same proportion. Medicinal plants represent an important therapeutic option in the studied community. The associated use of traditional and conventional medicines, besides the forms of knowledge transmission and conservation are discussed in this paper.
\end{abstract}

Key words: ethnobotany, popular medicine, Porto Alegre, local knowledge

\section{INTRODUÇÃO}

No Brasil, diferentes tradições terapêuticas contribuíram para a formação da medicina popular (Amorozo, 2004). Além da assimilação dos conhecimentos indígenas, as colaborações trazidas pelos escravos e imigrantes representaram papel importante para o surgimento de uma medicina popular rica e original, na qual a utilização de plantas medicinais ocupa lugar de destaque (Simões et al., 1995).

Atualmente, grande parte da população

Recebido para publicação em 18/06/2008

Aceito para publicação em 17/10/2008 
brasileira encontra nas plantas medicinais importante fonte de recurso terapêutico. Isso se deve a vários fatores, dentre os quais é possível destacar a crise econômica e o alto custo dos medicamentos industrializados, bem como, o difícil acesso da população à assistência médica. Aliada a essa situação verifica-se crescente tendência dos consumidores de utilizar "produtos naturais" (Di Stasi, 1996) e ainda o fato de muitas pessoas se renderem à facilidade de obtenção de plantas medicinais, as quais muitas vezes são cultivadas nos quintais de suas casas (Pilla et al., 2006).

Por outro lado, constatam-se perdas de conhecimento tradicional associado à biodiversidade brasileira e conseqüente diminuição do número de espécies empregadas para tratamento das enfermidades. Entre os fatores relacionados à perda do conhecimento sobre plantas medicinais no Brasil, encontram-se a redução das áreas naturais e a desvalorização dos saberes tradicionais pelas novas gerações, associados a crescente acesso à medicina convencional (Amorozo, 2002). Desta forma, tornase importante a realização de registros do conhecimento tradicional (Joshi \& Joshi, 2000), antes que as espécies e o conhecimento associado a elas sejam eliminados.

A valorização e registro do conhecimento tradicional sobre plantas medicinais é também objetivo de políticas públicas nacionais (Brasil, 2006) e estaduais (Rio Grande do Sul, 2001). Neste contexto, o presente trabalho se propõe a contribuir para o conhecimento da medicina popular do Rio Grande do Sul através de estudo etnobotânico das plantas medicinais utilizadas por especialistas locais no bairro do Lami, município de Porto Alegre, Rio Grande do Sul.

\section{MATERIAL E MÉTODO}

O estudo foi desenvolvido no bairro do Lami, situado no extremo sul da cidade de Porto Alegre, no Estado do Rio Grande do Sul, tendo como coordenadas aproximadas $30^{\circ} 15^{\prime} \mathrm{S}$ e $51^{\circ} 05^{\prime} \mathrm{W}$. Essa região da cidade, classificada como rural-ubana, caracteriza-se por apresentar "uma importante produção rural de hortigranjeiros desenvolvida em áreas que se intercalam com vários núcleos de ocupação urbana" (Hickel et al., 1999). Além da agricultura, destacam-se no bairro a pesca artesanal e o pequeno comércio.

Essa macrozona também é conhecida pelo patrimônio natural, constituído por morros e terras planas que se estendem até o Lago Guaíba. Os remanescentes florestais de Porto Alegre se localizam principalmente nessa região. Essas características, aliadas à presença da rara gimnosperma efedra
(Ephedra tweediana Fisch e C.A Mey), justificaram a criação de unidade de conservação municipal, a Reserva Biológica do Lami (RBL).

Apesar do relativo grau de urbanização, a comunidade do Lami ainda não dispõe de atendimento primário de saúde adequado. Não existe farmácia no bairro, ficando ao encargo do posto médico distribuir alguns dos medicamentos industrializados utilizados por esta comunidade. Quando existe a necessidade de se adquirir algum desses medicamentos, os moradores têm que se deslocar até a farmácia do bairro vizinho de Belém Novo, distante cerca de dez quilômetros.

Em 2000, foi criada no Bairro do Lami a Farmácia Caseira Comunitária Casa Verde. Trata-se de iniciativa de produção caseira de fitoterápicos, vinculada ao Movimento de Mulheres Camponesas (MMC) do Brasil e sediada na área de uso intensivo da Reserva Biológica do Lami (Casa Verde) até o ano de 2007, quando encerrou as atividades.

A partir de conversas informais realizadas com moradores da comunidade, foram selecionados inicialmente três especialistas. Um especialista, no contexto deste trabalho, pode ser definido como pessoa reconhecida pela comunidade por possuir profundo conhecimento acerca dos usos de plantas nativas e/ou introduzidas, manufaturar remédios e promover curas (Gazzaneo et al., 2005). Através do método bola-de-neve (Patton, 2001), no qual um informante selecionado sugeria o nome de outro(s) informante(s), foram escolhidos mais quatro especialistas, totalizando sete entrevistados. Este número representava, na ocasião do estudo, todos os especialistas em plantas medicinais reconhecidos pela maioria da comunidade.

Foram realizadas entrevistas semiestruturadas e gravações com esses sete informantes, os quais foram entrevistados em suas moradias, possibilitando o reconhecimento de espécies existentes nas hortas ou canteiros dos mesmos. Com alguns informantes, realizaram-se excursões guiadas para reconhecimento e/ou coleta de plantas.

Foram obtidos dados acerca da utilização das plantas, procedência, formas de preparo, parte utilizada, dose e contra-indicações. Para a obtenção desses dados, os entrevistados foram confrontados com situações potenciais de uso de espécies medicinais, conforme sugerido por Amorozo (1996).

As plantas citadas foram identificadas com o auxílio de lupas, literatura especializada e através de comparação com exsicatas do herbário "Instituto de Ciências Naturais" (ICN) do Departamento de Botânica da Universidade Federal do Rio Grande do Sul (UFRGS). Algumas espécies foram enviadas para especialistas para identificação e outras foram determinadas no local. Em alguns casos, foram realizadas entrevistas com fotografias, a fim de 
reconhecer espécies não disponíveis para coleta. As plantas coletadas foram herborizadas e as exsicatas foram incorporadas ao herbário ICN-UFRGS.

Os entrevistados foram deixados livres para se expressar dentro das próprias concepções de planta medicinal, o que no caso deste trabalho inclui as plantas utilizadas para simpatias, benzeduras, contra mau-olhado e olho grande, banhos de descarga e proteção. Para a realização das análises, as doenças foram categorizadas de acordo com a classificação do CID-10 - Classificação estatística internacional de doenças e problemas relacionados à saúde (Organização Mundial da Saúde, 2007).

Foram ainda calculados os índices de valor de uso (Rossato et al., 1999) e de concordância de uso (Amorozo \& Gély, 1988) para as espécies citadas. Também foi estimado o fator de Consenso entre Informantes $(\mathrm{FCl})$ para as diversas categorias de uso de plantas medicinais encontradas no estudo (Heinrich et al., 1998).

\section{RESULTADO E DISCUSSÃO}

\section{Uso das plantas}

Foi registrado o uso de 141 espécies com finalidade medicinal, sendo que a média do número de espécies utilizadas por informante foi de 89 plantas $(s=7,56)$. Em trabalhos realizados no Litoral Norte do Rio Grande do Sul, Marodin \& Baptista (2001) registraram o uso de 131 espécies a partir de entrevistas com 30 informantes. Já no município de Cruz Alta, também situado no RS, Garlet \& Irgang (2001) listaram a utilização de 189 espécies, mas neste caso o universo amostral foi de cerca de 150 pessoas.

TABELA1. Espécies medicinais que apresentaram os maiores índices de valor de uso (VU) na comunidade do Lami, Porto Alegre, RS.

\begin{tabular}{|c|c|c|c|c|}
\hline Nome vernacular & Nome científico & Origem & № de citações & VU \\
\hline Chá-de-bugre & Casearia sylvestris Swartz. & nativa & 13 & 1,86 \\
\hline Abacate & Persea americana Mill & exótica & 11 & 1,57 \\
\hline Guanxuma & Sida rhombifolia L. & nativa & 11 & 1,57 \\
\hline Marcela & Achyrocline satureioides DC. & nativa & 11 & 1,57 \\
\hline Pitangueira & Eugenia uniflora L. & nativa & 11 & 1,57 \\
\hline Poejo & Cunilla microcephala Benth. & nativa & 11 & 1,57 \\
\hline Carqueja & Baccharis articulata Pers. & nativa & 10 & 1,43 \\
\hline Funcho & Foeniculum vulgare Mill. & exótica & 10 & 1,43 \\
\hline Malva & Malva sp. & nativa & 10 & 1,43 \\
\hline Banana-do-mato & Bromelia antiacantha Bertol. & nativa & 9 & 1,29 \\
\hline Bergamota & Citrus reticulata Blanco & exótica & 9 & 1,29 \\
\hline Boldo sp.1 & Plectranthus barbatus Andr. & exótica & 9 & 1,29 \\
\hline Capim cidró & Cymbopogon citratus Stapf & exótica & 9 & 1,29 \\
\hline Cinamomo & Melia azedarach L. & exótica & 9 & 1,29 \\
\hline Gervão & Stachytarpheta cayennensis Schau & nativa & 9 & 1,29 \\
\hline Quebra-pedra & Phyllanthus sp. & nativa & 9 & 1,29 \\
\hline Sabugueiro & Sambucus australis Cham. Et Schlecht & exótica & 9 & 1,29 \\
\hline Chuchu & Sechium edule Sw. & exótica & 8 & 1,14 \\
\hline Erva-cidreira & Aloysia triphylla (L'Hér.) Britton & exótica & 8 & 1,14 \\
\hline Erva-de-bicho & Polygonum punctatum Elliot & nativa & 8 & 1,14 \\
\hline Eucalipto & Eucaliptus sp. & exótica & 8 & 1,14 \\
\hline Jurubeba & Solanum paniculatum L. & nativa & 8 & 1,14 \\
\hline Menstruz & Coronopus didymus (L.) Smith & nativa & 8 & 1,14 \\
\hline Romã & Punica granatum L. & exótica & 8 & 1,14 \\
\hline Salsaparrilha & Smilax campestris Griseb. & nativa & 8 & 1,14 \\
\hline Açoita-cavalo & Luehea divaricata Mart. & nativa & 7 & 1,00 \\
\hline Alecrim & Rosmarinus officinalis L. & nativa & 7 & 1,00 \\
\hline Arruda & Ruta sp. & exótica & 7 & 1,00 \\
\hline Cardo-santo & Cirsium vulgare (Savi) Ten. & exótica & 7 & 1,00 \\
\hline Cipó-mil-homens & Aristolochia triangularis Cham. & nativa & 7 & 1,00 \\
\hline Colírio & Commelina erecta $\mathrm{L}$. & nativa & 7 & 1,00 \\
\hline Fel-da-terra & Scoparia dulcis L. & nativa & 7 & 1,00 \\
\hline Goiabeira & Psidium guaiava L. & exótica & 7 & 1,00 \\
\hline Guaco & Mikania leavigata Sch. Bip. ex Baker & exótica & 7 & 1,00 \\
\hline Laranjeira & Citrus sinensis Osbeck & exótica & 7 & 1,00 \\
\hline Sete-sangrias & Cuphea carthaginensis Macbride & exótica & 7 & 1,00 \\
\hline Tansagem & Plantago sp & nativa & 7 & 1,00 \\
\hline
\end{tabular}


O elevado número de espécies medicinais conhecidas pelos especialistas da comunidade do Lami indica grande conhecimento dos mesmos sobre o tema em questão. Naturalmente, o número de plantas utilizadas pelos entrevistados no dia-a-dia é bem menor do que o conhecimento sobre a flora medicinal. Contudo, apesar de não utilizarem todas as plantas mencionadas, todos os informantes têm por hábito receitar plantas para parentes, amigos e às vezes desconhecidos que os procuram.

As espécies que apresentaram os maiores índices de valor de uso são apresentadas na Tabela 1. Trinta e sete espécies apresentaram valor de uso igual ou maior que um, sendo que o chá-de-bugre (Casearia sylvestris Swartz) foi a espécie com valor de uso (VU) mais elevado $(1,86)$. A seguir, com VU de 1,57 foram registradas as espécies abacate (Persea americana Mill), guanxuma (Sida rhombifolia L.), marcela (Achyrocline satureioides DC.), pitangueira (Eugenia uniflora L.) e poejo (Cunilla microcephala Benth.). Em geral, as plantas com maior VU encontradas neste trabalho correspondem às espécies comumente usadas no Brasil (Begossi et al., 2002; Hanazaki et al., 2006; Pilla et al., 2006; Negrelle et al., 2007).

Quando considerada a porcentagem de concordância quanto aos usos principais (CUP-Tabela 2), calculada para as espécies com os maiores VU, a ordem dos valores obtidos para cada espécie é alterada em relação aos apresentados na Tabela 1. As espécies que apresentaram valores máximo de CUP foram a banana-do-mato (Bromelia antiacantha

TABELA2. Percentagem de concordância em relação aos usos principais das espécies medicinais com maiores VU (índice de valor de uso) na comunidade do Lami, Porto Alegre (ICUE= informantes que citaram o uso da espécie; ICUP= número de informantes que citaram os usos principais; CUP= porcentagem de concordância quanto aos usos principais; $C U P c=$ CUP corrigida).

\begin{tabular}{|c|c|c|c|c|c|}
\hline Nome científico & Uso principal & ICUE & ICUP & CUP & CUPc \\
\hline Bromelia antiacantha Bertol. & tosse & 7 & 7 & 100,0 & 100,0 \\
\hline Cunilla microcephala Benth. & tosse & 7 & 7 & 100,0 & 100,0 \\
\hline Phyllanthus sp. & pedra nos rins & 7 & 7 & 100,0 & 100,0 \\
\hline Psidium guaiava $\mathrm{L}$. & diarréia & 7 & 7 & 100,0 & 100,0 \\
\hline Punica granatum $\mathrm{L}$. & diarréia & 7 & 7 & 100,0 & 100,0 \\
\hline Achyrocline satureioides DC. & dor de estômago & 7 & 6 & 85,7 & 85,7 \\
\hline Melia azedarach L. & ferida & 7 & 6 & 85,7 & 85,7 \\
\hline Mikania leavigata Sch. Bip. ex Baker & tosse & 6 & 6 & 100,0 & 85,7 \\
\hline Plectranthus barbatus Andr. & dores de estômago e fígado & 6 & 6 & 100,0 & 85,7 \\
\hline Sambucus australis Cham. Et Schlecht & sarampo & 6 & 6 & 100,0 & 85,7 \\
\hline Stachytarpheta cayennensis Schau & tosse & 6 & 6 & 100,0 & 85,7 \\
\hline Aloysia triphylla (L'Hér.) Britton & calmante & 7 & 5 & 71,4 & 71,4 \\
\hline Baccharis articulata Pers. & emagrecer & 7 & 5 & 71,4 & 71,4 \\
\hline Commelina erecta $\mathrm{L}$. & colírio & 5 & 5 & 100,0 & 71,4 \\
\hline Eugenia uniflora $\mathrm{L}$. & diarréia & 7 & 5 & 71,4 & 71,4 \\
\hline Foeniculum vulgare Mill. & gases & 6 & 5 & 83,3 & 71,4 \\
\hline Luehea divaricata Mart. & tosse & 6 & 5 & 83,3 & 71,4 \\
\hline Polygonum punctatum Elliot & hemorróidas & 6 & 5 & 83,3 & 71,4 \\
\hline Sechium edule Sw. & pressão alta & 6 & 5 & 83,3 & 71,4 \\
\hline Smilax campestris Griseb. & limpar o sangue & 6 & 5 & 83,3 & 71,4 \\
\hline Solanum paniculatum $\mathrm{L}$. & dor no fígado & 6 & 5 & 83,3 & 71,4 \\
\hline Casearia sylvestris Swartz. & afinar o sangue & 7 & 4 & 57,1 & 57,1 \\
\hline Cymbopogon citratus Stapf & calmante & 7 & 4 & 57,1 & 57,1 \\
\hline Citrus reticulata Blanco & tosse & 6 & 4 & 66,7 & 57,1 \\
\hline Malva sp. & inflamação & 6 & 4 & 66,7 & 57,1 \\
\hline Ruta sp. & proteção & 6 & 4 & 66,7 & 57,1 \\
\hline Coronopus didymus (L.) Smith & tosse e gripe & 5 & 3 & 60,0 & 42,9 \\
\hline Cirsium vulgare (Savi) Ten. & estômago & 7 & 3 & 42,9 & 42,9 \\
\hline Eucaliptus sp. & gripe & 6 & 3 & 50,0 & 42,9 \\
\hline Persea americana Mill & rins e para urinar & 6 & 3 & 50,0 & 42,9 \\
\hline Rosmarinus officinalis L. & proteção & 6 & 3 & 50,0 & 42,9 \\
\hline Scoparia dulcis L. & abrir o apetite & 7 & 3 & 42,9 & 42,9 \\
\hline Citrus sinensis Osbeck & gripe & 5 & 2 & 40,0 & 28,6 \\
\hline Cuphea carthaginensis Macbride & hemorróidas & 3 & 2 & 66,7 & 28,6 \\
\hline Plantago sp. & dor de garganta & 6 & 2 & 33,3 & 28,6 \\
\hline Sida rhombifolia L. & dor de barriga & 6 & 2 & 33,3 & 28,6 \\
\hline Aristolochia triangularis Cham. & não tem & 7 & 0 & 0,0 & 0,0 \\
\hline
\end{tabular}


Berthol.), o poejo (C. microcephala), o quebra-pedra (Phyllanthus sp.), a goiabeira (Psidium guaiava L.) e a romã (Punica granatum $\mathrm{L}$.). Também obtiveram altos valores de concordância $(85,7 \%)$ as espécies marcela (A. satureioides), cinamomo (Melia azedarach L.), guaco (Mikania leavigata Sch. Bip. ex Baker), boldo (Plectanthus barbatusAndr), sabugueiro (Sambucus australis Cham. et Schlecht) e gervão (Stachytarpheta cayennensis Schau).

Já espécies que apresentaram alto valor de uso como abacate (P.americana), chá de bugre (C.sylvestris), tansagem (Plantago sp.) e guanxuma (S. rhombifolia) obtiveram baixos valores de CUP, sendo que não houve consenso de uso para a espécie cipó-mil-homens (Aristolochia triangularis Cham.), utilizada por todos os informantes. Tais resultados não são contraditórios, uma vez que o VU tende a destacar as espécies com mais usos, ao passo que a CUP irá ressaltar o conhecimento compartilhado pelos informantes em relação ao uso das espécies. Por outro lado, a falta de consenso entre o uso das espécies também pode ser um indicativo de sua versatilidade. Negrelle \& Fornazzari (2007) registraram as espécies Plantago major L. e Aristolochia sp. associadas a um grande número de atividades terapêuticas em comunidades rurais do Paraná.

As famílias botânicas mais utilizadas pelos especialistas da comunidade do Lami são Asteraceae e Lamiaceae, seguidas de Myrtaceae e Rutaceae. Tais famílias são referidas como mais utilizadas em vários trabalhos com enfoque em plantas medicinais no Brasil (Hanazaki et al., 2000; Garlet \& Irgang, 2001;Amorozo, 2002; Gazzaneo etal., 2005; Negrelle et al.; 2007). Ao todo são utilizadas 63 famílias botânicas entre pteridófitas, mono e dicotiledôneas.

Constatou-se que $51 \%$ das plantas usadas como medicinais pelos informantes são nativas do Rio Grande do Sul enquanto que $49 \%$ são exóticas. Tal proporção de espécies nativas em relação às exóticas assemelha-se ao encontrados em estudos realizados no Rio Grande do Sul (Garlet \& Irgang, 2001) e nos demais estados do Brasil (Begossi et al. 2002; Almeida et al., 2006). Todavia, alguns autores apontam a predominância de espécies exóticas nas citações de uso medicinal em áreas de Mata Atlântica (Voeks, 1996; Pinto et al., 2006). De acordo com estes autores, as razões que poderiam explicar este padrão são complexas e estão relacionadas ao processo de colonização e à facilidade de cultivo das espécies exóticas ao redor das casas. De fato, grande parte das espécies exóticas citadas foi encontrada nas hortas e canteiros dos entrevistados. Na maioria das sociedades rurais brasileiras, quintais e pomares próximos à moradia desempenham importante função na manutenção de muitas espécies medicinais (Amorozo, 1996).

Por outro lado, o grande número de espécies nativas utilizadas provavelmente reflete a influência cultural dos índios Guarani, dos quais alguns dos informantes descendem. É provável que a alta diversidade de espécies medicinais conhecidas pelos especialistas entrevistados relacione-se com a troca de recursos genéticos durante os processos migratórios, seja entre agricultores europeus, seja entre migrantes e nativos brasileiros. Assim, a presença e utilização de espécies nativas e exóticas nesta comunidade é reflexo de seu dinamismo cultural, bem como das diferentes identidades que coexistem em seu interior.

No que se refere à forma de vida das plantas utilizadas, a maioria das espécies (45\%) são ervas, seguidas de árvores (20\%), arbustos (19\%) e arvoretas (9\%). Ainda são utilizadas lianas e hemiparasitas (4\%) e epífitas (3\%). A parte da planta predominantemente utilizada nas preparações é a folha (50\%). Também são utilizados frutos, cascas, flores e raízes e, em menor quantidade, sementes, resinas e látex. A predominância do uso de folhas é freqüente no Rio Grande do Sul (Garlet \& Irgang, 2001; Marodin \& Baptista, 2001), sendo que o uso de cascas e raízes é padrão mais comum em outras formações vegetais como o cerrado e a caatinga (Amorozo, 2002; Albuquerque \& Andrade, 2002), onde a disponibilidade de folhas pode ser limitada.

A principal forma de utilização de plantas medicinais é o chá, seguido de xaropes e alcoolaturas. O chá geralmente é preparado por infusão. Muitas plantas também são colocadas no chimarrão e tomadas diariamente. A maioria dos entrevistados ressaltou a importância de se preparar o chá sem ferver a planta, pois a fervura queimaria o "ser da erva". Esta consideração demonstra o conhecimento dos informantes acerca da possível perda de princípios ativos de diversas espécies durante o processo de decocção (Simões \& Spitzer, 1999; Souza \& Wiest, 2007). Uma exceção seria o preparo de chás a partir de cascas ou raízes onde seria possível ferver a planta por alguns minutos sem que ela perdesse as propriedades.

Os xaropes, preparados no inverno, são usados principalmente para tosse, gripe e bronquite. Já dentre as alcoolaturas, destaca-se uma mistura de cachaça com grande quantidade de ervas, chamada por todos os informantes de "arnique". O arnique, que é preparado para uso tópico, é usado em machucados, contusões, dores musculares, inflamações, dores reumáticas e outras enfermidades. Cada informante tem a sua "fórmula" de arnique, mas em geral são utilizadas as seguintes espécies: catinga-de-mulata (Tanacetum vulgare L.), abacate (P.americana), alcanflor (Artemisia camphorata Vill.), cipó-mil-homens (A. triangularis), confrei (Symphytum officinale L.), menstruz (Coronopus didymus (L.) Smith), angico (Parapiptadenia rigida (Benth) Brenan), 
alevante (Mentha piperita L.) e corticeira (Erythrina crista-galli L.).

Também foi registrado o emprego de algumas plantas potencialmente tóxicas, de acordo com Schenkel et al. (1999). Dentre estas se encontram o confrei (Symphytum officinale), a camaradinha (Lantana camara L.), a buchinha (Luffa sp.), a losna (Artemisia absinthium L.), a jurubeba (Solanum paniculatum L.), a babosa (Aloe arborescens Mill.) e uma das espécies conhecidas como chapéu-de-couro (Senecio bonariensis Hook. et Arn). Outras espécies tóxicas foram registradas, contudo os entrevistados raramente mencionaram contra-indicações das espécies por eles utilizadas. Nestes casos, buscouse um diálogo com os entrevistados, através da socialização de algumas informações acadêmicas sobre a possível toxicidade das espécies mencionadas.

\section{Tratamento das doenças e sistemas médicos utilizados pela comunidade}

De acordo com os resultados obtidos, o uso de plantas medicinais na região está predominantemente relacionado às afecções dos sistemas digestivo e respiratório, tendência encontrada em outros estudos realizados no Brasil (Hanazaki et al., 1996; Amorozo, 2002; Pilla et al., 2006; Negrelle \& Fornazzari, 2007). São empregadas 57 espécies para tratar as afecções do sistema digestivo e 50 espécies para as doenças do sistema respiratório (Tabela 3). Já para o tratamento de sintomas, sinais e anormalidades clínicas tais como febre e dor são utilizadas 39 espécies. Outros sistemas cujas doenças são tratadas com grande variedade de espécies são o genitourinário (30 espécies) e o circulatório (29 espécies).

Ressalta-se que, além de ser empregado o maior número de espécies nas doenças dos sistemas digestivo e respiratório, estes também apresentaram elevados valores do fator de consenso dos informantes (FCl) acerca das plantas utilizadas, fato também registrado por Almeida et al. (2006). O valor máximo de FCI foi encontrado para as doenças do olho, o que é justificado pelo fato de ser empregada uma única espécie para tratamento de tais doenças (Commelina erecta L.). Além dos sistemas já mencionados, altos valores de $\mathrm{FCl}$ foram registrados para o tratamento de "doenças culturais" (mau-olhado, quebrante, entre outras) e doenças infecciosas e parasitárias. Estes valores indicam que, no caso destas doenças, existe um critério de seleção bem definido para escolha de plantas pela comunidade e/ou que ocorre troca de informação entre os especialistas locais (Gazzaneo et al., 2005). Tais resultados assemelham-se aos de Heinrich (1998), que encontrou valores altos de FCl para o caso de doenças gastrointestinais e para as "síndromes culturais".

Algumas categorias apresentaram baixos valores de consenso entre informantes. Não foi encontrado consenso entre os informantes na categoria "injúria, envenenamento e causas externas", na qual são utilizadas nove diferentes espécies e entre as doenças do sangue e sistema imunológico foi registrado $\mathrm{FCl}$ de 0,17 . Este resultado pode indicar ausência de rígidos critérios seletivos na escolha de

TABELA3. Fator de Consenso entre Informantes $(\mathrm{FCl})$ para diversas categorias de uso de plantas medicinais na comunidade do Lami, Porto Alegre, RS.

\begin{tabular}{lccccc}
\hline Categoria de doença & Espécies & $\begin{array}{c}\text { \% total de } \\
\text { espécies }\end{array}$ & $\begin{array}{c}\text { Citações } \\
\text { de uso }\end{array}$ & $\begin{array}{c}\% \text { todas as } \\
\text { citações }\end{array}$ & $\mathbf{F C l}$ \\
\hline Doenças do olho & 1 & 0,71 & 5 & 0,74 & 1,00 \\
Doenças do sistema digestivo & 57 & 40,71 & 177 & 26,11 & 0,68 \\
Doenças do sistema respiratório & 50 & 35,71 & 140 & 20,65 & 0,65 \\
"Doenças culturais" & 19 & 13,57 & 42 & 6,19 & 0,56 \\
Doenças infecciosas e parasitárias & 7 & 5,00 & 14 & 2,06 & 0,54 \\
Doenças do sistema circulatório & 29 & 20,71 & 55 & 8,11 & 0,48 \\
Doenças do sistema genitourinário & 30 & 21,43 & 56 & 8,26 & 0,47 \\
Doenças da pele e tecidos sub-cutâneos & 25 & 17,86 & 41 & 6,05 & 0,40 \\
Sintomas, sinais, anormalidades clínicas & 39 & 27,86 & 60 & 8,85 & 0,36 \\
Doenças do sistema nervoso & 19 & 13,57 & 29 & 4,28 & 0,36 \\
Doenças do sistema músculo-esquelético & 14 & 10,00 & 21 & 3,10 & 0,35 \\
Doenças endócrinas, nutricionais e metabólicas & 12 & 8,57 & 18 & 2,65 & 0,35 \\
Doenças do ouvido e processos mastóides & 3 & 2,14 & 4 & 0,59 & 0,33 \\
Doenças do sangue e sistema imunológico & 6 & 4,29 & 7 & 1,03 & 0,17 \\
Injúria, envenenamento e causas externas & 9 & 6,43 & 9 & 1,33 & 0,00
\end{tabular}


plantas para tratar destas enfermidades.

A fim de contextualizar melhor os resultados encontrados, foram obtidos dados acerca das doenças apresentadas por pacientes atendidos pelos médicos do posto de saúde do Lami, no período de duração deste trabalho. Segundo esses dados, as afecções mais freqüentes neste período foram as dos sistemas respiratório e circulatório, seguidas de dores musculares e verminoses.

É possível notar que existe similaridade entre o uso de plantas medicinais e o registro de doenças realizado pelo posto de saúde, o que pode ser um indicativo das doenças mais comuns sofridas pelos membros da comunidade do Lami. Contudo, a baixa incidência de doenças do aparelho digestivo no posto de saúde pode ser um indicativo de que essas são tratadas com plantas medicinais. Em outros casos, como o das doenças respiratórias, há uma provável interação entre os tipos de tratamento.

Segundo relato dos especialistas entrevistados, muitas pessoas que sofrem de bronquite asmática no Lami recorrem a chás e xaropes caseiros, nebulizações, broncodilatadores e benzeduras. Outra prática comum registrada nesse trabalho foi a dissolução de comprimidos analgésicos e antitérmicos em infusões de plantas utilizadas para tratar resfriados e gripes. Portanto, o tratamento oferecido pela medicina tradicional se sobrepõe à medicina convencional no caso das infecções respiratórias, fato observado também por Vandebroek et al. (2008).

Os resultados das entrevistas também apontam para a grande utilização de plantas mágicorituais. Esses dados demonstram a grande influência das religiões afro-brasileiras, especialmente da umbanda, nas concepções de saúde e doença dos entrevistados. Essas concepções, por sua vez, determinam quais práticas são utilizadas para a cura das enfermidades. Entre as práticas utilizadas destacam-se os banhos de descarga, as benzeduras para diversas doenças e as plantas usadas para proteção e contra mau-olhado.

A possibilidade de cura das enfermidades é um dos fatores que atrai muitas pessoas para as religiões afro-brasileiras. A escolha do tipo de tratamento adotado está fortemente relacionada à crença espiritual/religiosa. Mas não é só a religião que determina se o tratamento ficará ao encargo do sistema oficial de saúde ou de especialistas religiosos. No caso do bairro do Lami, as pessoas têm difícil acesso à medicina convencional e farmácias. Esse fator pode estar influenciando a comunidade a optar pelo tratamento com especialistas religiosos, independentemente de suas crenças.

No local estudado, esses especialistas religiosos são as benzedeiras. Essas, além de receitarem plantas medicinais, rezam e benzem. Essa prática muito comum, tanto nos grandes centros como nos pequenos e em zonas rurais, remonta a tempos muito antigos (Arruda Camargo, 1998). Desta forma, na comunidade do Lami os diferentes sistemas médicos não estão isolados, ocorrendo típico sincretismo terapêutico. $O$ caso das doenças respiratórias ilustra a integração entre explicações provenientes de diferentes sistemas de conhecimento. Este sincretismo terapêutico, onde vários sistemas médicos interagem na busca das pessoas pela cura das doenças, demonstra que as pessoas geralmente buscam se beneficiar de todas as oportunidades de tratamento disponíveis a elas (Amorozo, 2004).

\section{Transmissão do conhecimento sobre plantas medicinais}

A transmissão oral é a principal forma pela qual o conhecimento sobre plantas medicinais vem sendo transmitido no bairro do Lami. De acordo com os entrevistados, seu aprendizado sobre o tema ocorreu de pai/mãe ou avô/avó para filho/a. Entretanto, a maioria deles destacou a perda de conhecimento sobre o assunto na localidade e o desinteresse atual por parte dos mais jovens em aprender sobre o uso das plantas medicinais. Este tipo de transmissão de conhecimento é diretamente dependente da integridade familiar e da ausência de pressões migratórias que podem levar os jovens para fora da comunidade. Quando isto não ocorre, a conseqüência pode ser a perda de conhecimento através do falecimento dos indivíduos mais velhos (Negrelle \& Fornazzari, 2007). Amorozo (1996) enfatiza o papel da educação formal como intensificadora deste quadro, pois ao retirar os jovens do convívio com os mais velhos durante grande parte do tempo, ela acaba fomentando o desinteresse por esses saberes.

Os entrevistados também destacaram a importância de se coletar as ervas, especialmente as para benzeduras, na mata virgem. Contudo, os mesmos destacaram a dificuldade que tem sido encontrar algumas espécies em função da diminuição de sua área de ocorrência. A transformação de ecossistemas locais através da expansão das áreas sujeitas a atividades econômicas humanas tem exercido severas limitações no acesso e disponibilidade de tipos específicos de plantas usadas para fins medicinais (Alves \& Rosa, 2007). A redução da ocorrência das espécies medicinais também acarreta na perda do conhecimento associado a tais recursos genéticos, o qual foi acumulado historicamente e transmitido através de inúmeras gerações.

Além da transmissão vertical (entre diferentes gerações), também é importante considerar a transmissão horizontal entre os sujeitos de uma 
geração. Na comunidade do Lami, essa transmissão se dá principalmente entre vizinhos que trocam mudas de plantas e receitas caseiras. Essa troca leva à incorporação de novas plantas por parte dos sujeitos envolvidos nela. Além disso, reforçalaços sociais e contribui paraa manutenção desse conhecimento nacomunidade.

Neste contexto, uma das alternativas encontradas pela comunidade foi a organização de uma farmácia caseira comunitária. Neste espaço de socialização e troca de saberes, reuniam-se semanalmente integrantes da comunidade do Lami, juntamente com representantes do meio acadêmico, a fim de "ajudar as pessoas a prevenir e tratar doenças de forma natural, através de conversas, percepções, orientações, amizade e, por último, uso de elixires, pomadas, tinturas e xaropes" (Printes, 2002). Estas atividades ocorriam em um espaço denominado "Casa Verde", zona de uso intensivo da Reserva Biológica do Lami, e estavam de acordo com o plano de manejo desta unidade de conservação (Printes, 2002).

Entre os objetivos do grupo estavam: "preservar o conhecimento tradicional e as plantas nativas da região e também suprir a carência de alternativas para a melhoria da saúde da comunidade do Lami, a qual não dispõe de nenhum tipo de farmácia e nem tampouco condições financeiras para adquirir medicamentos industrializados" (Printes, 2002).

Tais objetivos estavam em consonância com as políticas públicas existentes na área de plantas medicinais. Dentre as diretrizes elencadas na Política Nacional de Plantas Medicinais e Fitoterápicos (Brasil, 2006) encontram-se a promoção e o reconhecimento das práticas populares de uso de plantas medicinais e remédios caseiros.

Já no âmbito estadual, a Política Intersetorial de Plantas Medicinais (Rio Grande do Sul, 2001) tem como objetivo a criação de mecanismos e instrumentos de proteção, resgate e valorização da cultura tradicional e popular sobre as plantas medicinais na saúde humana, animal e vegetal. Esta política ainda tem por finalidade o incentivo e apoio à construção de propostas alternativas e democráticas sobre plantas medicinais, como forma de valorizar, socializar e potencializar a criatividade das comunidades.

Entretanto, apesar da existência destas políticas públicas, as quais validam experiências como a das Farmácias Caseiras Comunitárias, constata-se que na prática ainda há um longo caminho a trilhar em busca do reconhecimento do conhecimento tradicional, bem como, da conservação destes saberes. O encerramento das atividades da Farmácia Caseira Comunitária Casa Verde (FCC Casa Verde) é emblemático desta situação. Apesar de estar em consonância com o plano de manejo da Reserva Biológica do Lami, a atual administração desta unidade impediu a continuidade das atividades da FCC, não reconhecendo a importância do conhecimento tradicional associado à biodiversidade e rompendo um dos principais elos entre a Reserva e a comunidade local. Tal fato, aliado às exigências de adequação do trabalho impostas pela Secretaria Municipal de Saúde de Porto Alegre, acabaram por inviabilizar esta iniciativa popular.

Entende-se que espaços como os das farmácias caseiras sejam oportunos para resgatar, valorizar e transformar o conhecimento sobre plantas medicinais. Em muitos casos, sabe-se que é necessária uma reorientação do trabalho realizado nestes locais, a fim de garantir o uso seguro das plantas medicinais. Esta reorientação deve se dar a partir da integração dos conhecimentos populares aos resultados de pesquisas acadêmicas, bem como de orientações advindas de profissionais de saúde com formação em fitoterapia. Para tanto, é fundamental a existência de "espaços de locução", nos quais deve ser enfatizada a dialogicidade entre os portadores de diferentes saberes.

No que se refere à comunidade estudada, conclui-se que as plantas medicinais representam uma importante alternativa terapêutica e que devem ser pensadas estratégias e novos espaços onde o conhecimento sobre plantas medicinais, especialmente o dos especialistas locais, seja valorizado e resgatado, evitando a erosão cultural que ocorre em todo o país.

\section{AGRADECIMENTO}

À equipe da Reserva Biológica do Lami, especialmente ao funcionário Cláudio Fraga pelos ensinamentos sobre plantas medicinais e ao exadministrador da unidade Rodrigo Cambará Printes pelo exemplo profissional, incentivo e apoio ao trabalho. À Prof ${ }^{a}$ Mara Ritter pelas sugestões no texto, ao Biólogo Marco Polo Laufer pelo auxílio com as planilhas e à Maria de Lourdes Duarte (Rafinha) pelas visitas à comunidade do Lami. Os autores agradecem especialmente aos integrantes da Farmácia Caseira Comunitária Casa Verde e a todos/as os/as entrevistados/as, pela disponibilidade e saberes compartilhados.

\section{REFERÊNCIA}

ALBUQUERQUE, U.P.; ANDRADE, L.H.C. Uso de recursos vegetais da caatinga: o caso do agreste do Estado de Pernambuco (Nordeste do Brasil). Interciencia, v.27, p.336-45, 2002. 
ALMEIDA, C.F.C.B. et al. Medicinal plants popularly used in the Xingó region Journal of Ethnobiology and Ethnomedicine, v.2, n.15, 2006. Disponível em <http:// www.ethnobiomed.com/content/2/1/15>. Acesso em: 15 fev. 2008.

ALVES, R.R.N.; ROSA, I.M.L. Biodiversity, traditional medicine and public health: where do they meet? Journal of Ethnobiology and Ethnomedicine, v.3, n.14, 2007. Disponível em: <http://www.ethnobiomed.com/content/ 3/1/14>. Acesso em: 15 fev. 2008.

AMOROZO, M.C.M.; GÉLY, A. Uso de plantas medicinais por caboclos do Baixo Amazonas, Barcarena, PA, Brasil. Boletim Museu Paraense Emílio Goeldi, v.4, n.1, p.47131, 1988.

AMOROZO, M.C.M. Abordagem etnobotânica na pesquisa de plantas medicinais. In: DI STASI, L.C. (Ed.) Plantas medicinais: arte e ciência. Um guia de estudo interdisciplinar. São Paulo: UNESP, 1996. p.47-68.

AMOROZO, M.C.M. Uso e diversidade de plantas medicinais em Santo Antonio do Leverger, MT, Brasil. Acta Botanica Brasilica, v.16, n.2, p.189-203, 2002. AMOROZO, M.C.M. Pluralistic medical settings and medicinal plant use in rural communities, Mato Grosso, Brazil. Journal of Ethnobiology, v.24, n.1, p.139-61, 2004. ARRUDA CAMARGO, M.T.L. Plantas Medicinais e rituais afro-brasileiros. II: estudo etnofarmacobotânico. São Paulo: Ícone, 1998. 232p.

BEGOSSI, A.; HANAZAKI, N.; TAMASHIRO, J.Y. Medicinal plants and the Atlantic Forest (Brazil): knowledge, use and conservation. Human Ecology, v.30, p.281-99, 2002. BRASIL. Decreto № 5.813, de 22 de Junho de 2006. Que aprova a Política Nacional de Plantas Medicinais e Fitoterápicos e dá outras providências. Disponível em: <http://www.planalto.gov.br/CCIVIL/decreto/D5813.htm>. Acesso em: 7 nov. 2007.

DISTASI, L.C. Arte, ciência e magia. In: DISTASI, L.C. (Ed.). Plantas medicinais: arte e ciência. Um guia de estudo interdisciplinar. São Paulo: UNESP, 1996. p.15-21.

GARLET, T.M.B.; IRGANG, B.E. Plantas medicinais utilizadas na medicina por mulheres trabalhadoras rurais de Cruz Alta, Rio Grande do Sul, Brasil. Revista Brasileira de Plantas Medicinais, v.4, n.1, p.9-18, 2001. GAZZANEO, L.R.S; LUCENA, R.F.P.L.; ALBUQUERQUE, U.P. Knowledge and use of medicinal plants by local specialists in an region of Atlantic Forest in the state of Pernambuco (Northeastern Brazil). Journal of Ethnobiology and Ethnomedicine, v.1, n.9, 2005. Disponível em: <http://www.ethnobiomed.com/Content/ 1/1/9>. Acesso em: 13 nov. 2007.

HANAZAKI, N.; LEITÃO FILHO, H.F.; BEGOSSI, A. Uso de recursos na Mata Atlântica: o caso da Ponta do Almada. Interciencia, v.21, n.6, p.268-76, 1996.

HANAZAKI, N. et al. Diversity of plant uses in two Caiçara communities from Atlantic Forest coast, Brazil. Biodiversity and Conservation, v.9, p.597-615, 2000. HANAZAKI, N.; SOUZA, V.C.; RODRIGUES, R.R. Ethnobotany of rural people from the boundaries of Carlos Botelho State Park, São Paulo State, Brazil. Acta Botanica Brasilica, v.20, n.4, p.899-909, 2006.

HEINRICH, M. et al. Medicinal plants in Mexico: healers' consensus and cultural importance. Social Science and Medicine, v.47, n.11, p.1859-71, 1998.

HICKEL, H.T. et al. Cidade rural-urbana. In: MENEGAT,
R. (Ed.). Atlas ambiental de Porto Alegre. Porto Alegre: Ed. UFRGS, 1999. p.118.

JOSHI, A.R.; JOSHI, K. Indigenous knowledge and uses of medicinal plants by local communities of the Kali Gandaki Watershed Area, Nepal. Journal of Ethnopharmacology, v.73, p.175-83, 2000.

MARODIN, S.M.; BAPTISTA, L.R.M. O uso de plantas com fins medicinais no município de Dom Pedro de Alcântara, Rio Grande do Sul, Brasil. Revista Brasileira de Plantas Medicinais, v.4, n.1, p.57-68, 2001.

NEGRELLE, R.R.B. et al. Estudo etnobotânico junto à Unidade Saúde da Família Nossa Senhora dos Navegantes: subsídios para o estabelecimento de programa de fitoterápicos na Rede Básica de Saúde do Município de Cascavel (Paraná). Revista Brasileira de Plantas Medicinais, v.9, n.3, p.6-22, 2007.

NEGRELLE, R.R.B.; FORNAZZARI, K.R.C. Estudo etnobotânico em duas comunidades rurais (Limeira e Ribeirão Grande) de Guaratuba (Paraná, Brasil). Revista Brasileira de Plantas Medicinais, v.9, n.2, p.36-54, 2007. ORGANIZAÇÃO MUNDIAL DE SAÚDE. International Statistical Classification of Diseases and Related Health Problems. 2007. Disponível em: <http://www.who. int/classifications/apps/icd/icd10online >. Acesso em: 27 jan. 2008.

PATTON, M.Q. Qualitative evaluation and research methods. 3.ed. California: Sage Publications, 2001. $688 p$.

PILLA, M.A.C.;AMOROZO, M.C.M.; FURLAN, A. Obtenção e uso das plantas medicinais no distrito de Martim Francisco, Município de Mogi-Mirim, SP, Brasil. Acta Botanica Brasilica, v.20, n.4, p.789-802, 2006.

PINTO, E.P.P.; AMOROZO, M.C.M.; FURLAN. A. Conhecimento popular sobre plantas medicinais em comunidades rurais de mata atlântica - Itacaré, BA, Brasil. Acta Botanica Brasilica, v.20, n.4, p.751-62, 2006. PRINTES. R.C. Plano de manejo participativo da Reserva Biológica do Lami. Porto Alegre: SMAM, 2002. 133p.

RIO GRANDE DO SUL. Política intersetorial de plantas medicinais para o Rio Grande do Sul. 2001. Disponível em <http://www.sct.rs.gov.br/programas/fito-rs/politica \%20intersetorial.htm>. Acesso em: 7 dez. 2005.

ROSSATO, S.C.; LEITÃO-FILHO, H.F.; BEGOSSI, A. Ethnobotany of caiçaras of the Atlantic Forest Coast (Brazil). Economic Botany, v.53, n.4, p.387-95, 1999. SCHENKEL, E.P. et al. Plantas tóxicas. In: SIMÕES, C.M.O. et al. (Ed.). Farmacognosia: da planta ao medicamento. Porto Alegre: Ed.UFSC/Ed.UFRGS, 1999. p.755-88.

SIMÕES, C.M.O. et al. Plantas da medicina popular no Rio Grande do Sul. 4.ed. Porto Alegre: Ed. UFRGS, 1995.174p.

SIMÕES, C.M.O.; SPITZER, V. Óleos voláteis. In: SIMÕES, C.M.O. et al. (Ed.). Farmacognosia: da planta ao medicamento. Porto Alegre: Ed.UFSC/Ed.UFRGS, 1999. p.387-416.

SOUZA, A.A.; WIEST, J.M. Atividade anti-bacteriana de Aloysia gratissima (Gill et Hook) Tronc. (garupá, erva santa), usada na medicina tradicional no Rio Grande do Sul - Brasil. Revista Brasileira de Plantas Medicinais v.9, n.3, p.23-9, 2007.

VANDERBROEK, I. et al. Comparison of health conditions treated with traditional and biomedical health care in a 
Quechua community in rural Bolivia. Journal of Ethnobiology and Ethnomedicine, v.4, p.1, 2008. Disponível em: <http://www.ethnobiomed.com/content/
4/1/1>. Acesso em: 2 mar. 2008.

VOEKS, R.A. Tropical forest healers and habitat preference. Economic Botany, v.50, n.4, p.381-400, 1996. 